\title{
Pulsar Death at an Advanced Age
}

\author{
Jonathan Arons \\ Department of Astronomy, Department of Physics and Theoretical \\ Astrophysics Center, University of California at Berkeley, 601 Campbell \\ Hall, Berkeley, CA 94720-3411, USA arons@astroplasma.berkeley.edu
}

\begin{abstract}
I summarize the theory of acceleration of non-neutral particle beams by starvation electric fields along the polar magnetic field lines of rotation powered pulsars, including the effect of dragging of inertial frames which dominates the acceleration of a space charge limited beam. I apply these results to a new calculation of the radio pulsar death line, under the hypotheses that pulsar "death" corresponds to cessation of pair creation over the magnetic poles and that the magnetic field has a locally dipolar topology. The frame dragging effect in star centered dipole geometry does improve comparison of the theory with observation, but an unacceptably large conflict between obsrvation and theory still persists. Offsetting the dipole improves the comparison, but a fully satisfactory theory requires incorporating magnetic conversion of inverse Compton gamma rays, created by scattering thermal photons from the surface of old neutron stars $\left(t>10^{8}\right.$ years) kept warm $\left(T \geq 10^{5} \mathrm{~K}\right)$ by friction between the rotating core and the crust. The result is a "death valley" for pulsars; offsets of the dipole center from the stellar center in the oldest stars $\sim(0.7-0.8) R_{*}$ suffice. The resulting theory predicted the existence of rotation powered pulsars with these advanced ages, a prediction confirmed by the recent discovery that PSR J2144-3933 actually has a rotation period of 8.5 seconds.
\end{abstract}

\section{Introduction}

Radio emission from Rotation Powered Pulsars (RPPs) probably has its origin in the relativistic outflow of electron-positron pairs along the polar magnetic field lines of a dipole magnetic field frozen into the rotating neutron star (e.g., Arons 1992, Meszaros 1992). The evidence for dipole magnetic fields comes primarily from the electromagnetic theory of RPP spindown energy losses, which occur at the rate $\dot{E}_{R}=k \mu^{2} \Omega_{*}^{4} / c^{3}=-I \Omega_{*} \dot{\Omega}_{*}$ (Dyson 1971, Arons 1979,1992 ). Here $\mu$ is the magnetic moment, $\Omega_{*}=2 \pi / P, P$ is the rotation period, and $k$ is a function of any other parameters of significance, with magnitude on the order of unity. In the vacuum theory (Deutsch 1955), $k=(2 / 3) \sin ^{2} i$, with $i$ the angle between the magnetic moment and the angular velocity. Theoretical work on the torques due to conduction currents steming back to Goldreich and Julian (1969), coupled to the approximate independence of spindown torques from observationally estimated values of $i$ (Lyne and Manchester 1988), suggest that in reality $k$ does 
not substantially depend on $i$. In the subsequent discussion, I assume $k=4 / 9$, the average of the vacuum value over the sphere. Application of this EM energy loss rate to the observations of RPPs' periods and period derivatives yields $\mu \sim 10^{30}$ cgs for "normal" RPPs, and $\mu \sim 10^{27}$ cgs for millisecond RPPs.

The electromagnetic torque interpretation of pulsar spindown constrains only the exterior dipole moment of the magnetic field. However, Rankin (1990) and Kramer et al. (1998) have presented strong evidence in favor of a low altitude $\left(r \approx R_{*}\right.$ ) dipole geometry for the site of the core component of pulsar radio emission. Arons (1993) gave evidence that spun up millisecond pulsars must have substantially dipolar fields at low altitute.

If electron-positron pair creation above the polar caps is important for radio emission, all observed pulsars must lie in the region of $P-\dot{P}$ space where polar cap acceleration has sufficient vigor to lead to copious pair production. Yet, to date, all internally consistent theories of polar cap pair creation have required hypothesizing a large scale (e.g., quadrupole) component of the magnetic field with strength comparable to that of the dipole (Ruderman and Sutherland 1975, Arons and Scharlemann 1979, Barnard and Arons 1982, Gurevich and Istomin 1985). Such strong magnetic anomalies contradict the evidence in favor of an apparently dipolar low altitude geometry; the alteration of the magnetic geometry also ruins the internal consistency of many models' electrodynamics.

Here I describe a low altitude polar cap acceleration theory which successfully associates pulsar "death" with the cessation of pair creation in an offset dipole low altitude magnetic field. The basic acceleration physics is that of a space charge limited relativistic particle beam accelerated along the field lines by the starvation electric field, as in the Arons and Scharlemann theory, but with the additional effect of inertial frame dragging, first pointed out by Muslimov and Tsygan (1990, 1992) and by Beskin (1990). If the dipole's center is offset from the stellar center, the magnetic field at one pole becomes substantially stronger than it would be if the same magnetic dipole were star centered. The location of an individual pulsar's pair creation death depends on the magnitude of the offset, thus yielding a "death valley" (Chen and Ruderman 1993) for the whole pulsar population. Finally, if thermal photon emission at temperature $T \sim 10^{5} \mathrm{~K}$ continues to great age ( $t>10^{8}$ years), as is is expected in neutron star models with late time heating due to friction between the crust and core, the theory with dipole offsets predicts and accounts for pulsars with very long periods and great age.

\section{Polar Acceleration}

Study of polar cap relativstic particle acceleration in the 1970's had led to the conclusion that acceleration of a space charge limited particle beam from the stellar surface with energy/particle high enough to emit magnetically convertible curvature gamma rays occurs because of curvature of the magnetic field (Scharlemann et al. 1978, Arons and Scharlemann 1979). In a curved $B$ field, matching of the beam charge density to the Goldreich-Julian density occurs only at the surface. Along field lines which curve toward the rotation axis ("favorably curved" field lines), the beam fails to short out the vacuum above the surface, Therefore, particles accelerate along $B$ through a potential drop 
$\Delta \Phi_{\|}=\Delta \Phi_{S A F} \approx \Phi_{\text {pole }}\left(R_{*} / \rho_{B}\right) \sim 10^{-2} P^{-1 / 2} \Phi_{\text {pole. }}$. The numerical value assumes field lines have dipolar radius of curvature $\rho_{B} \sim \sqrt{R_{*} c / \Omega_{*}}$. Here $\Phi_{\text {pole }} \equiv$ $\Omega_{*}^{2} \mu / c^{2}=1.09 \times 10^{13}\left(I_{45} / k\right)^{1 / 2}\left(\dot{P}_{15} / P^{3}\right)^{1 / 2}$ Volts, with $\dot{P}_{15} \equiv \dot{P} / 10^{-15} \mathrm{~s} / \mathrm{s}$ and $I_{45}=I / 10^{45} \mathrm{~g}-\mathrm{cm}^{2}$. Particles drop through the potential $\Delta \Phi_{S A F}$ over a length $L_{\|} \sim R_{*}$.

Curvature gamma rays have typical energy $\varepsilon_{c} \sim\left(\hbar c / \rho_{B}\right)\left(e \Delta \Phi_{\|} / m c^{2}\right)^{3} \propto$ $\Phi_{\text {pole }}^{3} / \rho_{B}^{4}$, while the optical depth for pair creation, due to one photon conversion of gamma rays emitted by electrons (orpositrons) can be shown to be $\tau=$ $\Lambda \exp \left[-a\left(m c^{2} / \varepsilon_{c}\right)\left(B_{q} / B_{*}\right)\left(\rho_{B} / R_{*}\right)\right]$ (Arons and Scharlemann 1979, Luo 1996, Bjornsson 1996), where $a$ is a pure number (typically $\sim 30$ ) and $\Lambda$ is a combination of the basic parameters which is quite large $(\ln \Lambda \sim 20)$. A reasonable theoretical definition of the death line is $\tau=1$. Using $B_{*}=2\left(\Phi_{\text {pole }} / R_{*}\right)\left(c / \Omega_{*} R_{*}\right)^{2}$, $\Delta \Phi_{S A F}$ and setting $\tau$ equal to unity yields the death line, expressed as $\Phi_{\text {death }}(P)$ such that stars $\Phi_{\text {pole }}<\Phi_{\text {death }}$ do not make pairs. This death line, appears as the dashed line in Figure 1. This figure shows clearly that the large dynamic range in $\Phi_{\text {pole }}, P$ space made available by the cataloging of millisecond pulsars falsifies this theory, even if one invokes non-dipolar radii of curvature to move the position of the death line vertically in the diagram - the scaling with period flatly disagrees with the shape of the boundary of pulsar radio emission in the $\Phi_{\text {pole }}, P$ diagram. These results imply either that something else governs the low altitude acceleration which leads to pair creation, or that pair creation is not important to radio emission.

Muslimov and Tsygan $(1990,1992)$ uncovered a previously overlooked effect on the acceleration of the non-neutral beam from the stellar surface. Stellar rotation drags the inertial frame into rotation, at the angular velocity $\omega_{L T}=$ $\left(2 G I / R_{*}^{2} c^{2}\right) \Omega_{*}\left(R_{*} / r\right)^{3}$. Therefore, the electric field required to bring a charged particle into corotation is $\mathbf{E}_{c o}=-(1 / c)\left[\left(\Omega_{*}-\omega_{L T}\right) \times \mathbf{r}\right] \times \mathbf{B}$; the magnetic field rotates with respect to local inertial space, not inertial space at infinity. The charge density required to support this local corotation electric field is $\eta_{R}=-\left[\left(\boldsymbol{\Omega}_{*}-\omega_{L T}\right) \cdot \mathbf{B}\right] / 2 \pi c=-\left[\boldsymbol{\Omega}_{*} \cdot \mathbf{B} / 2 \pi c\right]\left[1-\kappa_{g}\left(R_{*} / r\right)^{3}\right]$, where $\kappa_{g}=$ $2 G I / R_{*}^{3} c^{2}=0.17\left(I_{45} / R_{10}^{3}\right)$. Relativistic space charge limited flow from the surface has a beam charge density $\eta_{b}=-\left(\Omega_{*} \cdot \mathrm{B}_{*} / 2 \pi c\right)\left(1-\kappa_{g}\right)\left(B / B_{*}\right)$. Above the surface, this charge density is too small to short out $E_{\|}$on all polar field lines, not just the favorably curved part of a polar flux tube, thus providing a theoretical basis for polar cap acceleration models to be in accord with the observed rough symmetry of radio emission with respect to the magnetic axis (Lyne and Manchester 1988). One can graphically describe this general relativistic origin of electrical starvation as the consequence of the field lines rotating faster with respect to inertial space as the radius increases, at the angular speed $\Omega_{*}-\omega_{L T}(r)=\Omega_{*}\left[1-\kappa_{g}\left(R_{*} / r\right)^{3}\right]$. The constraint of relativistic flow along $B$ allows the beam to provide only a charge density sufficient to support corotation at the angular speed $\Omega_{*}\left(1-\kappa_{g}\right)$. The difference not surprisingly leads to an accelerating potential drop $\Delta \Phi_{\|} \approx \kappa_{g} \Phi_{\text {pole }}\left[1-\left(R_{*} / r\right)^{3}\right]$. For normal $(P \sim 1 \mathrm{sec})$ pulsars with dipole fields, the effect of dragging of inertial frames on the beam's acceleration yields curvature gamma ray energies 1000 times greater than occur in the Arons and Scharlemann pair creation theory; for MSPs, the theories yield 
comparable results, although of course the symmetry of the beam with respect to the magnetic axis differs.
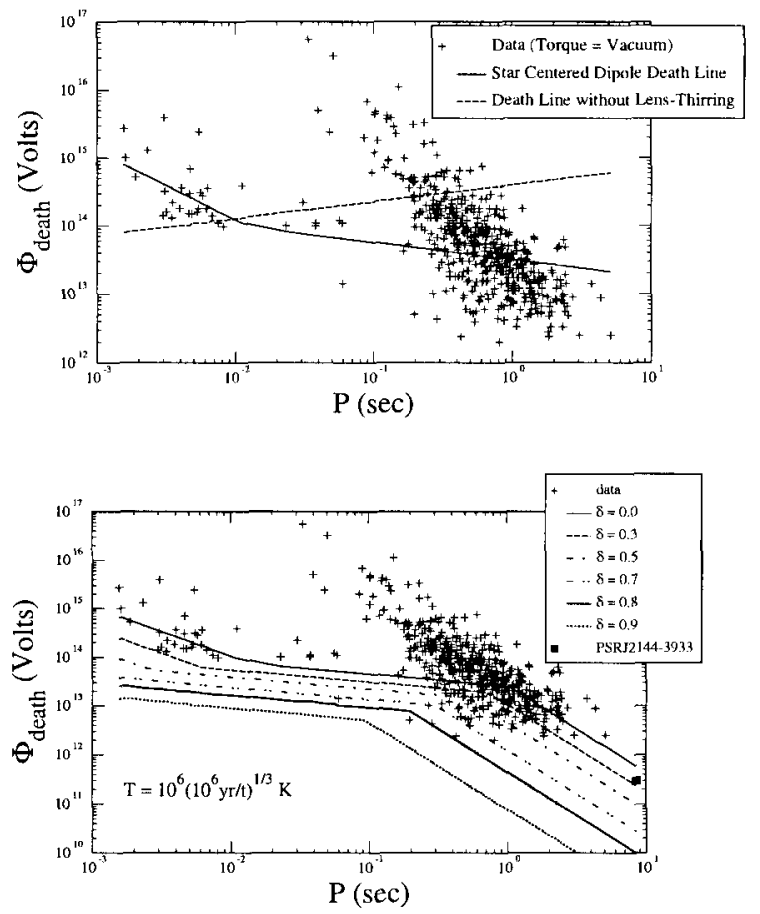

Figure 1. Upper panel: Pair creation Death Lines for Star Centered Dipoles. The solid line gives the result for curvature gamma ray emission and and one photon magnetic conversion, when the beam acceleration model incorporates the effect of inertial frame dragging. The dashed line show the result for the same geometry and the same gamma. ray emission and absorption physics, but with inertial frame dragging neglected in the particle acceleration theory. Lower panel: Death Valley for an offset vector parallel to the dipole moment, with both curvature and resonant inverse Compton emission as gamma ray sources, and with stellar temperature kept high by internal heating. The filled square shows the location of the recently discovered long period pulsar J2144-3933.

\section{Death Lines and Death Valley}

When curvature emission is the only source of gamma rays, the death line for a star centered dipole is shown in Figure 1. Dragging of inertial frames clearly improves the agreement between the boundary of pair activity in the $\Phi-P$ diagram and the region where pulsars occur, but the discrepancy is still too 
large - something else is missing. If the field geometry must be locally dipolar at low altitude, then the only ingredients still not included are 1) offset of the dipole from the stellar center and 2) additional gamma ray emission and absorption processes. The simplest dipole offset has the magnetic field of a point dipole, with the center of the dipole displaced from the stellar center by an offset vector parallel to $\mu$. This has the effect of increasing the magnetic field at one pole to strength $B_{*}=2 \mu /\left(R_{*}-\delta\right)^{3}$, with a resulting drastic increase in the gamma ray opacity, while leaving the accelerating potential unaltered. The results with curvature radiation as the only gamma ray emission process show that dipole offsets do allow such a curvature radiation theory of pulsar death to survive the challenge of modern observations, although at the price of displacements of the dipole center from the stellar center comparable to moving the dipole's center to the base of the crust. Such a model still does not account for PSR J2144-3933 (Young et al. 1999), however.

Curvature emission is not the only means of converting beam energy to gamma rays. ROSAT observations have revealed the long sought thermal Xrays from neutron star surfaces (Becker and Trümper 1997). Resonant Compton scattering creates magnetically convertible gamma rays at a spatial rate $\left(d N_{\gamma} / d s\right)_{r C} \propto T / \Gamma^{2}$, (e.g., Luo 1996) where $T$ is the temperature of the cooling neutron star (polar cap heating is unimportant in death valley) and $\Gamma=$ $e \Phi / m_{ \pm} c^{2}$ is the Lorentz factor of an electron or positron in the beam. Compton scattering thus can become a significant source of gamma rays in stars with small accelerating potentials. Compton scattering thus may contribute significantly for stars with low overall voltage.

This expectation is correct, if internal heating (e.g., Umeda et al. 1993) keeps the surface temperature above $10^{5} \mathrm{~K}$ at spindown ages in excess of $10^{7.5}$ years. In this case, resonant Compton scattering of thermal photons by a polar electron beam does extend death valley to include all the observed pulsars, with offsets required in the lowest voltage RPPs of order $60 \%$ to $70 \%$, as is shown in the lower panel of Figure 1. Note that this theory predicted (Arons 1998) the existence of RPPs with large periods $(P>5$ seconds) and unusually low voltage $\Phi_{\text {pole }}<10^{12.5}$ Volts [great age, $t=170\left(10^{12} V / \Phi_{\text {pole }}\right)^{2}\left(10^{s} / P\right)^{2}$ Myr.]

Indeed, recent observations (Young et al. 1999) have found a a $P=8.51$ second pulsar, PSR J2144-3933, which falls below the death line for a star centered dipole (even including Compton scattering), but lies comfortably within death valley, when both Compton scatering and dipole offsets are included.

\section{Conclusion}

I have shown that polar pair creation based on acceleration of a steadily flowing, space charged limited non-neutral beam in a locally dipolar magnetic geometry at low altitude is consistent with pulsar radio emission throughout the $P-P$ diagram, provided 1) the effect of dragging of inertial frames is included in estimates of the starvation electric field; 2) the dipole center is strongly offset from the stellar center in older stars, perhaps as much as $0.7-0.8 R_{*}$; and 3 ) inverse Compton emission of thermal photons from a neutron star cooling slower than exponentially at ages in excess of $10^{6}$ years plays an important role in the emission of magnetically convertible gamma rays. The development of new 
diagnostics of the low altitude magneic field, gamma ray observations sensitive to low altitude emission, and optical and UV observations of thermal emission from old, nearby RPPs will eventually provide tests of these ideas.

Acknowledgments. My research on pulsars is supported in part by NSF grant AST 9528271 and NASA grant NAG 5-3073, and in part by the generosity of California's taxpayers.

\section{References}

Arons, J. 1979, Space Sci. Rev., 24, 437

1992, Proc. IAU Colloq. No. 128, 'The Magnetospheric Structure and Emission Mechanisms of Raio Pulsars', T.H. Hankins, J.M. Rankin and J. A. Gil, eds. (Zielona Gora: Pedagogical University Press), 56 1993, Ap.J., 408, 160 1998, in 'Neutron Stars and Pulsars: Thirty Years After the Discovery', N. Shibazaki et al., eds (Tokyo: Universal Academy Press), 339 (astro$\mathrm{ph} / 9802198)$

Arons, J., and Scharlemann, E.T. 1979, Ap.J., 231, 854

Beskin, V.S. 1990, Pis'ma Ast. Zh., 16, 665 (Sov. Ast. - Letters, 16)

Barnard, J.J., and Arons, J. 1982, Ap.J., 254, 713

Becker, W., and Trümper, J. 1997, A\&A, 326, 682

Bjornsson, C.-I. 1996, Ap.J., 471, 321

Chen, K., and Ruderman, M.A. 1993, Ap.J., 402, 264

Deutsch, A.J. 1955, Ann. Ap., 18, 1

Dyson, F.J. 1971, "Neutron Stars and Pulsars: Fermi Lectures 1970" (Rome: Acadmia Nazionale dei Lincei), 25-26

Goldreich, P. and Julian, W. 1969, Ap.J., 157, 869

Gurevich, A.V., and Istomin, Ya.N. 1985, Zh.Eksp.Teor.Fiz., 89,3 (Soviet Physis - JETP, 62, 1)

Kramer, M., et al. 1998, Ap.J., 501, 270

Luo, Q. 1996, Ap.J., 468, 338

Lyne, A.G., and Manchester, R.N. 1988, MNRAS, 234, 477

Meszaros, P. 1992, "High Energy Radiation from Magnetized Neutron Stars" (Chicago: University of Chicago Press)

Muslimov, A., and Tsygan, A.I. 1990, Ast. Zh., 67, 263 (Soviet Ast., 34, 133) 1992, MNRAS, 255, 61

Rankin, J.M. 1990, Ap.J., 352, 247

Ruderman, M.A., and Sutherland, P.G. 1975, Ap.J., 196, 51

Scharlemann, E.T., Arons, J., and Fawley, W.M. 1978, Ap.J., 222, 297

Umeda, H., et al. 1993, Ap.J., 408, 186

Young, M. et al. 1999, Nature, 400, 848 